\title{
Follicular T-Cell Lymphoma with Concomitant Lennert Lymphoma
}

\author{
Seungkyu Choi · Jai-Hyang Go \\ Department of Pathology, Dankook University College of Medicine, Cheonan, Korea
}

Follicular T-cell lymphoma (FTCL) has recently been re-categorized by the World Health Organization as a member of the angioimmunoblastic T-cell lymphomas (AITLs) and other nodal lymphomas of $\mathrm{T}$ follicular helper (TFH) cell origin from peripheral T-cell lymphoma (PTCL) not otherwise specified (NOS). Lennert lymphoma (LeL) is considered to be a variant of PTCL, NOS. ${ }^{2}$ The coexistence of LeL with FTCL has not previously been reported. Herein, we describe an unusual case of FTCL with associated LeL, suggesting a possible relationship between these two entities as part of the TFH-derived lymphomas.

\section{CASE REPORT}

A 64-year-old male patient was admitted with a neck mass that had been present for 1 month. Computed tomography showed multiple enlarged lymph nodes along the left side of the neck from level I to V. An excisional biopsy of the neck mass was performed.

The architecture of the excised lymph nodes was completely effaced by multiple nodules of ill-defined small $\mathrm{IgD}+$ mantle zone B cells (Fig. 1A, B). Within the B cell nodules, several aggregates of small to medium lymphoid cells with round nuclei and clear cytoplasm were present (Fig. 1C). Double immunostaining for BOB-1 and CD10 was performed. Most BOB-1 (-) atypical tumor cells were positive for $\mathrm{CD} 3, \mathrm{CD} 4, \mathrm{CD} 10, \mathrm{PD}-1$, and BCL6 (Fig. 1D). These findings are compatible with FTCL with the growth pattern of progressive transformation of germinal center (PTGC). Focally, the area of LeL was intimately admixed

Corresponding Author

Jai-Hyang Go, MD

Department of Pathology, Dankook University College of Medicine, 119 Dandae-ro,

Dongnam-gu, Cheonan 31116, Korea

Tel: +82-41-550-6972, Fax: +82-41-561-9127, E-mail: cyjy555@hanmail.net

Received: March 20, 2018 Revised: April 13, 2018

Accepted: April 16, 2018 with typical FTCL components (Fig. 2A). LeL components showed evenly distributed prominent clusters of epithelioid cells, which were surrounded by small to medium atypical cells (Fig. 2B). In double immunostaining for BOB-1 and CD10, many BOB-1 (-) atypical tumor cells were positive for CD10 (Fig. 2C, D), PD-1, and BCL6. No follicular dendritic cell (FDC) hyperplasia was noted in either the FTCL or LeL components. Analysis of T-cell $\gamma$ gene (TCR- $\gamma$ ) rearrangement studies using BIOMED-2 -based polymerase chain reaction demonstrated clonal peaks at the same location generated using a DNA template from either the FTCL (Fig. 3A) or LeL components (Fig. 3B).

The Institutional Review Board of Dankook University Hospital (2018-03-007) approved this case report, and informed consent was waived.

\section{DISCUSSION}

We describe an unusual case of FTCL with associated LeL, suggesting a possible relationship between these two entities.

FTCL is a lymph node-based neoplasm of TFH cells with a predominantly follicular growth pattern and lacking characteristic features of AITL, such as proliferation of high endothelial venules or extrafollicular FDCs. Two distinct growth patterns are recognized: one that mimics follicular lymphoma and one that mimics PTGC. ${ }^{1}$ While FTCL and AITL have some overlapping clinical and pathologic features, ${ }^{2}$ FTCL seems to represent a peculiar stage of AITL in which neoplastic cells remain located within B-cell follicles. ${ }^{2}$ In a limited number of cases in which consecutive biopsies from different times were studied, change in morphology from FTCL to typical AITL, or vice versa, has been observed. ${ }^{1}$ Some cases of AITL relapse with FTCL and rare cases of FTCL with coexistent AITL have been reported. ${ }^{3}$ These findings suggest that these two entities may constitute different morphologic representations of the same biological process. ${ }^{1}$ 

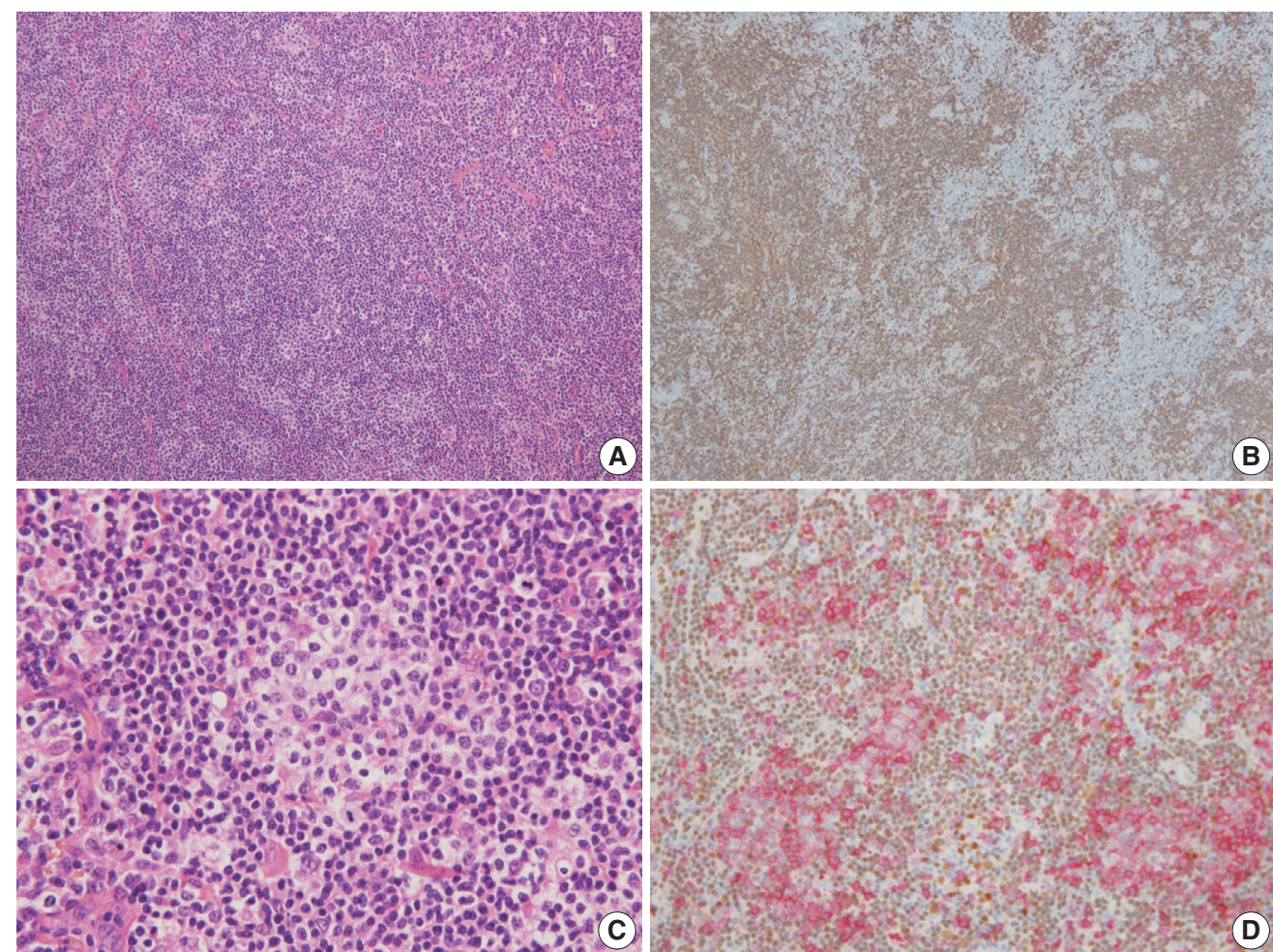

Fig. 1. (A) Lymph node architecture is totally effaced by multiple ill-defined nodules of small lymphocytes. (B) Most cells in the nodules are positive for CD20. (C) Within B-cell nodules, aggregates of small to medium atypical lymphoid cells with round nuclei and clear cytoplasm are present. (D) In double immunostaining for BOB-1 in brown (DAB) and CD10 in red (AEC), BOB-1 (-) tumor cells are diffusely positive for CD10.
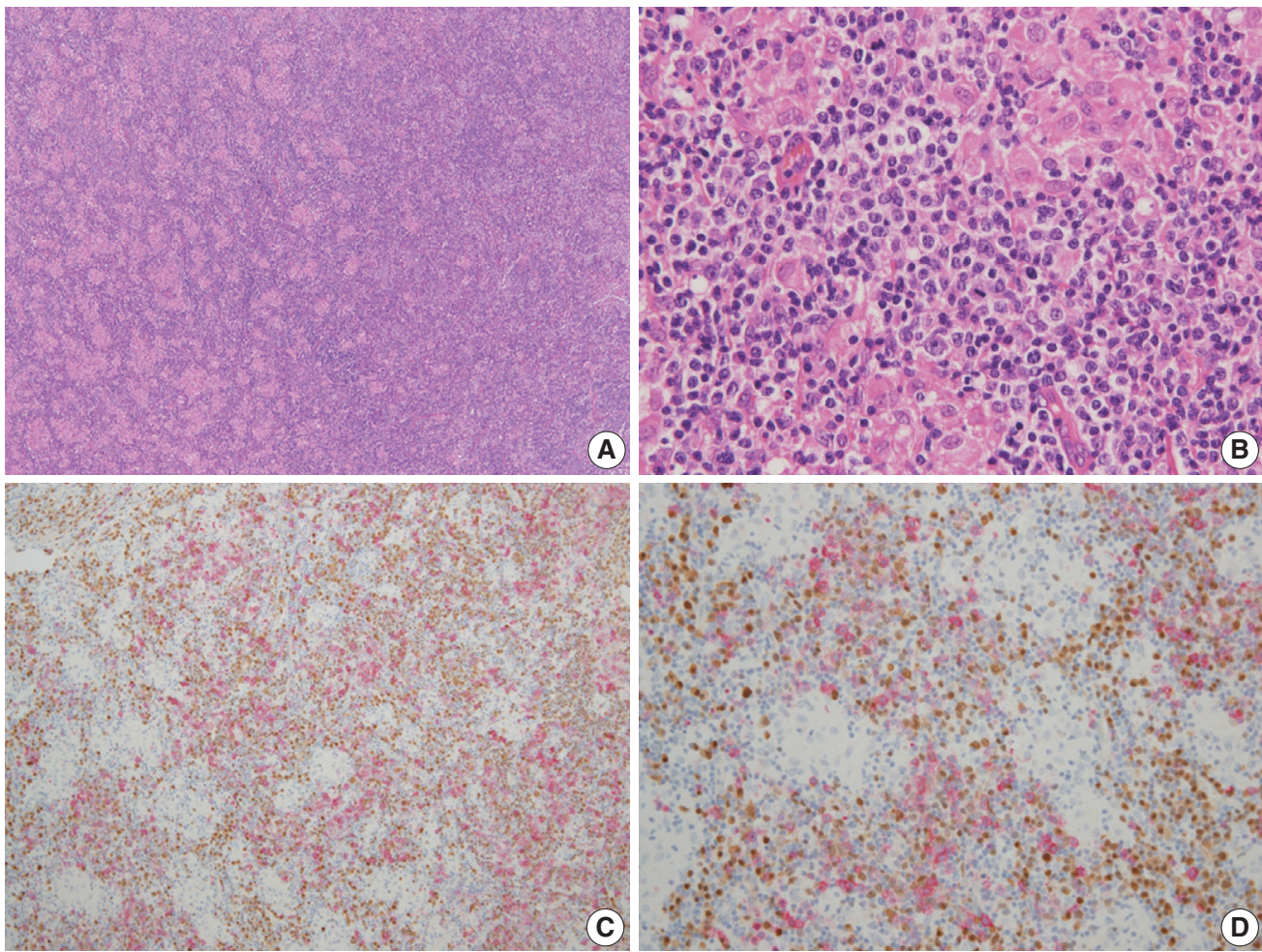

Fig. 2. (A, B) Prominent clusters of epithelioid cells surrounded by small to medium atypical cells are focally present. (C, D) In double immunostaining for BOB-1 in brown (DAB) and CD10 in red (AEC), many BOB-1 (-) tumor cells are positive for CD10. 

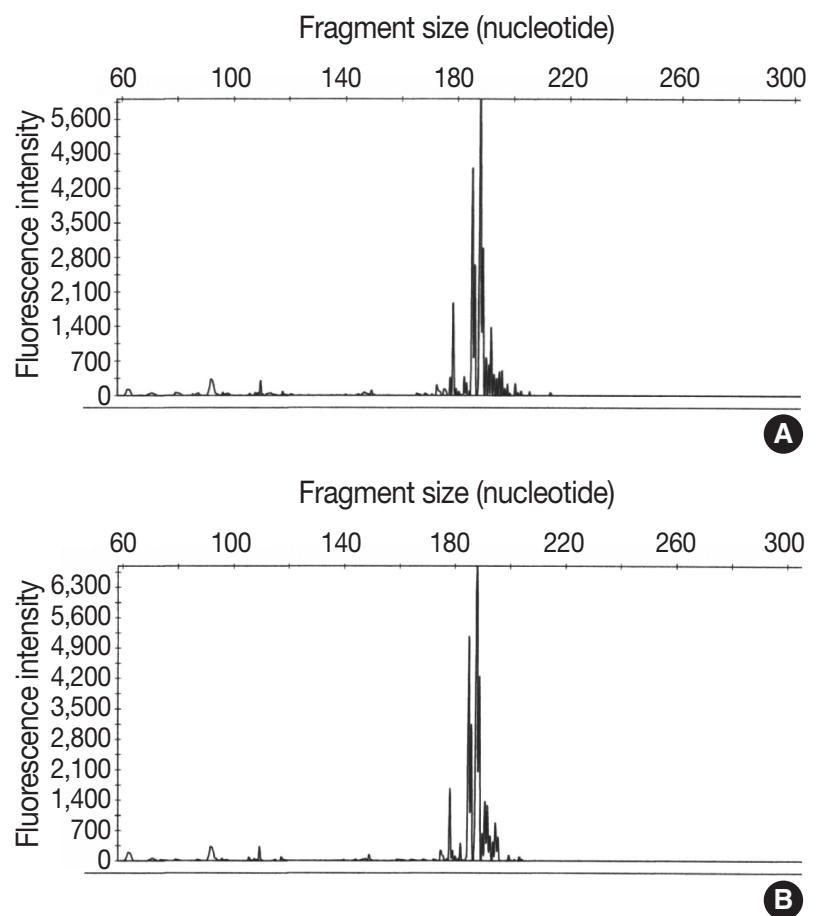

Fig. 3. Analysis of T-cell $\gamma$ gene rearrangement studies using BIOMED-2-based polymerase chain reaction shows clonal peaks at the same location in both follicular T-cell lymphoma (A) and Lennert lymphoma components (B).

LeL is a rare variant of PTCL, NOS characterized by a prominent reactive infiltrate of epithelioid histiocytes that are distributed singly or, more typically, in small clusters. The tumor cells are usually small with slightly irregular nuclear contours. ${ }^{4,5}$ Diagnosis of these tumors is usually based on pure morphology, and the differential diagnosis includes other epithelioid cell-rich lymphomas, especially AITL. ${ }^{6}$ Some cases of AITL are considered to have histopathologic features that overlap with those of LeL. However, distinct diagnostic criteria for immunohistochemical properties or histopathologic features and definitive criteria for distinguishing between AITL and LeL have not yet been established. ${ }^{6}$

The TFH cell surface markers, PD-1, CXCL13, CD10, and BCL6, are frequently and characteristically expressed in AITL. ${ }^{6}$ However, individual TFH cell markers can be expressed by other T-cell subsets, ${ }^{3}$ and are detected in $20 \%$ to $41 \%$ of PTCL-NOS. Recently, a significant number of LeL cases positive for these markers were described. ${ }^{6}$ TFH marker-positive cases had a worse prognosis than marker-negative cases and showed a similar prognosis to AITL, although many clinicopathologic features differed significantly between TFH marker-positive LeL and AITL. TFH marker-positive LeL could be a subset of AITL because it exhibits some of the features of AITL, such as high expression of TFH markers, and a similar prognosis. ${ }^{6}$

In the present case, the LeL component was intimately admixed with the FTCL component, and the TFH markers CD10, PD1, and BCL6 were comparably positive for these two types of tumors. Taken together, these findings support the suggestion that LeL might be appropriately included under the category of TFHderived lymphomas in addition to AITL and FTCL.

\section{Conflicts of Interest}

No potential conflict of interest relevant to this article was reported.

\section{REFERENCES}

1. Dogan A, Gaulard P, Jaffe ES, Muller-Hermelink HK, de Leval L. Angioimmunoblastic T-cell lymphoma and other nodal lymphomas of $\mathrm{T}$ follicular helper (TFH) cell origin. In: Swerdlow SH, Campo E, Harris NL, et al., eds. WHO classification of tumors of hematopoietic and lymphoid tissues. Revised 4th ed. Lyon: IARC Press, 2017; 407-12.

2. Huang Y, Moreau A, Dupuis J, et al. Peripheral T-cell lymphomas with a follicular growth pattern are derived from follicular helper $\mathrm{T}$ cells (TFH) and may show overlapping features with angioimmunoblastic T-cell lymphomas. Am J Surg Pathol 2009; 33: 682-90.

3. Hu S, Young KH, Konoplev SN, Medeiros LJ. Follicular T-cell lymphoma: a member of an emerging family of follicular helper T-cell derived T-cell lymphomas. Hum Pathol 2012; 43: 1789-98.

4. Jaffe E, Arber DA, Campo E, Harris NL, Quintanilla-Fend L. Hematopathology. 2nd ed. Philadelphia: Elsevier, 2017; 64405.

5. Hartmann S, Agostinelli C, Klapper W, et al. Revising the historical collection of epithelioid cell-rich lymphomas of the Kiel Lymph Node Registry: what is Lennert's lymphoma nowadays? Histopathology 2011; 59: 1173-82.

6. Kurita D, Miyoshi H, Yoshida N, et al. A clinicopathologic study of Lennert lymphoma and possible prognostic factors: the importance of follicular helper T-cell markers and the association with angioimmunoblastic T-cell lymphoma. Am J Surg Pathol 2016; 40: 1249-60.

7. Pileri SA, Weisenburger DD, Sng I, et al. Peripheral T-cell lymphoma, NOS. In: Swerdlow SH, Campo E, Harris NL, et al., eds. WHO classification of tumors of hematopoietic and lymphoid tissues. Revised 4th ed. Lyon: IARC Press, 2017; 403-6. 